\title{
Cateter ventriculoperitoneal migrado para o escroto através de hérnia inguinal - Relato de caso
}

\author{
Eduardo Procaci da Cunha1, Sérgio Moreira1, Mário Gomes", \\ Isabel Mesquita², Vítor Simões², Ernesto de Carvalho' \\ Centro Hospitalar do Porto, Porto, Portugal.
}

\section{RESUMO}

A derivação ventriculoperitoneal é atualmente o método mais utilizado no tratamento da hidrocefalia comunicante. As complicações mais comuns são a disfunção do shunt e infecções. Este relato de caso tem como objetivo apresentar um doente com idas recorrentes ao serviço de urgência por agitação psicomotora e dor abdominal, e cujo cateter distal previamente desconectado encontrava-se alojado no interior do saco escrotal através de hérnia inguinoescrotal. A formação de hérnia inguinal com migração de cateter é um evento raro no adulto, sendo mais comum na criança, possivelmente pela maior taxa de patência do processo vaginal e aumento da pressão intra-abdominal. No momento em que este artigo foi redigido, não foi encontrado caso semelhante na literatura.

\section{PALAVRAS-CHAVE}

Derivação ventriculoperitoneal, hidrocefalia, hérnia inguinal.

\section{ABSTRACT}

Ventriculoperitoneal shunt migrated to scrotum through inguinal hernia - Case report Ventriculoperitoneal shunt is the most used method for the treatment of communicating hydrocephalus. Common complications include shunt dysfunction and infection. This case report aims to present a patient, with recurrent visits to the emergency department for abdominal pain and agitation, and whose distal catheter previously disconnected, was housed inside the scrotum through a scrotal inguinal hernia. The association of inguinal hernia and catheter migration is uncommon in adults, being more frequent in infants possibly because of the higher rate of patent processus vaginalis and increased abdominal pressure. No similar case was found in the literature by the time this article has been written.

\section{KEYWORDS}

Ventriculoperitoneal shunt, hydrocephalus, hernia inguinal. 


\section{Introdução}

A derivação ventriculoperitoneal (DVP) é o procedimento de eleição no tratamento de diversas causas de hidrocefalia. Embora seja rara, a migração do cateter abdominal é possível e está descrita em diferentes localizações como o trato gastrointestinal, ${ }^{1,2}$ parede abdominal, ${ }^{3}$ vagina $^{4}$ e mediastino. ${ }^{5} \mathrm{~A}$ migração do cateter para o escroto é um evento raro na criança, ${ }^{6,7}$ tendo como fatores de risco a idade ${ }^{8}$ e o aumento da pressão intra-abdominal ${ }^{9,10} \mathrm{e}$ da patência do processo vaginal. No momento da redação deste artigo, não foi encontrado nenhum caso descrito no adulto.

Neste relato é descrito um caso em que um adulto sem história prévia de hérnia inguinal recorre repetidamente ao serviço de urgência por agitação psicomotora e dor abdominal.

\section{Descrição do caso}

Homem de 55 anos com antecedentes de traumatismo cranioencefálico (TCE) grave em agosto de 2007, submetido inicialmente à drenagem de hematoma subdural agudo e posteriormente à DVP por hidrocefalia comunicante pós-TCE. Neurologicamente, o doente ficou dependente de terceiros em suas necessidades diárias, com discurso pouco fluente e parcialmente orientado, com marcha independente e ligeira hemiparesia esquerda sequelar. Em maio de 2010, necessitou de revisão distal do shunt por desconexão entre a válvula e o cateter distal, que se encontrava migrado para o interior da cavidade abdominal. Foi inserido um novo cateter, e o antigo ficou alojado na cavidade peritoneal.

Ao longo do ano de 2012, o doente recorreu quatro vezes ao serviço de urgência por estar progressivamente menos colaborante, com episódios de agitação psicomotora e referência verbal à dor em diversas regiões do tórax e abdome. A elaboração de um diagnóstico foi dificultada pela incapacidade do doente de fornecer uma anamnese adequada ou de colaborar durante o exame físico. Nesse contexto, realizou diversos exames complementares sem ter sido possível a concretização de um diagnóstico final. Em setembro de 2012, num novo retorno ao serviço de urgência, passou a referir gestualmente dor na região inguinal direita. Nesse contexto, foi objetivada tumefação da região inguinal direita (Figura 1) compatível com hérnia inguinal, e à palpação do saco escrotal, notou-se a presença de estrutura tubular com consistência semelhante a de um cateter ventriculoperitoneal. Para além do estado de agitação, o restante do exame neurológico era sobre- ponível ao estado habitual do doente e não havia sinais de complicação da hérnia inguinal.

$\mathrm{O}$ estudo foi complementado com radiografias abdominal e pélvica (Figura 2), que confirmaram a presença de cateter ventriculoperitoneal no interior do saco escrotal. Após interpretação das imagens, concluiu-se que o cateter alojado no saco herniário seria aquele previamente desconectado e que a ponta do cateter conectado à válvula estaria no interior do canal inguinal direito.

O doente foi operado na semana seguinte, conjuntamente com cirurgia geral. Foi removido o saco herniário que continha o cateter (desconectado) enrolado em seu interior, bem como a ponta do outro cateter funcionante (Figuras 3 e 4). Procedeu-se ao reposicionamento do cateter no interior da cavidade abdominal e hernioplastia com colocação de prótese Progrip autoadesiva.

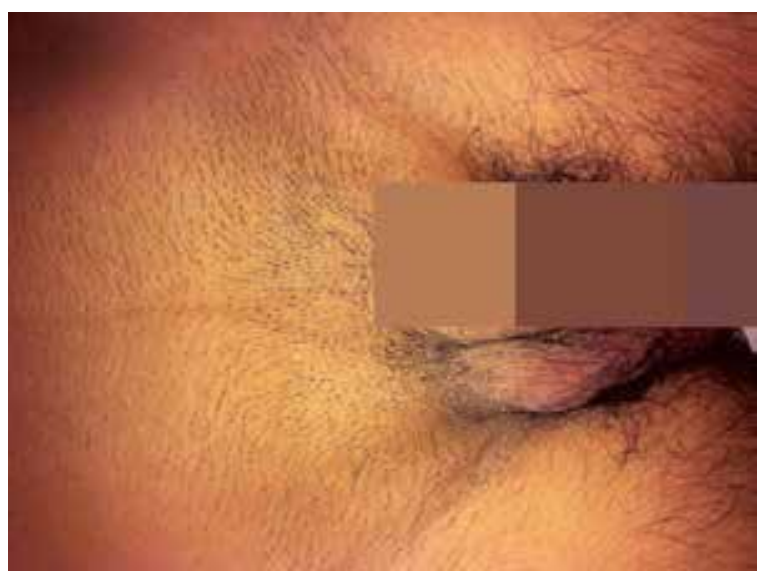

Figura 1 - Tumefação inguino-escrotal à direita. À palpação era possível notar estrutura tubular enrolada correspondente ao cateter abdominal.

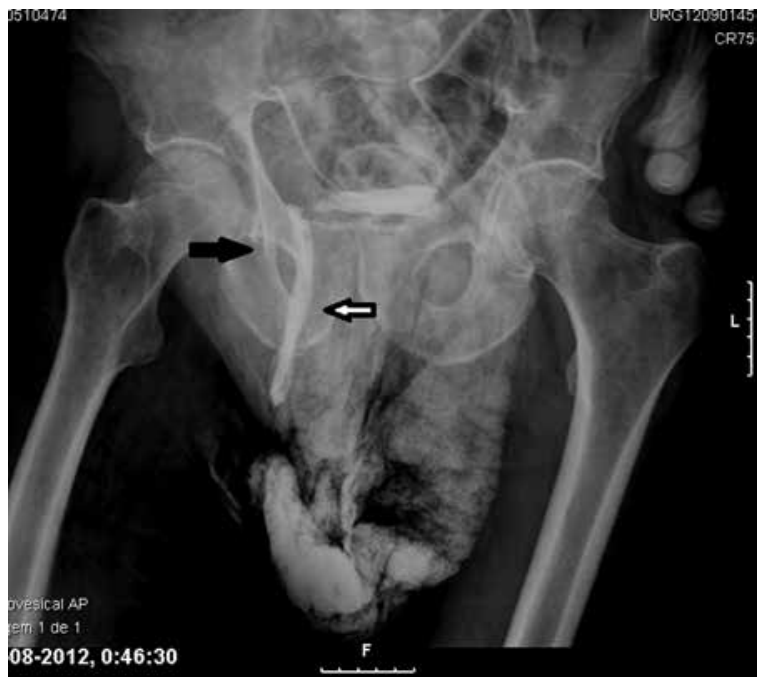

Figura 2 - A seta negra mostra a ponta do cateter abdominal funcionante. A seta branca aponta o cateter abdominal desconectado, alojado pela região inguinal até o saco escrotal. 
O doente teve alta hospitalar no dia seguinte com melhora da dor referida à fossa ilíaca direita e resolução da agitação psicomotora. Após seis meses de follow-up, a esposa refere melhora significativa do comportamento do doente, sem novos episódios de agitação ou passagem pelo serviço de urgência.

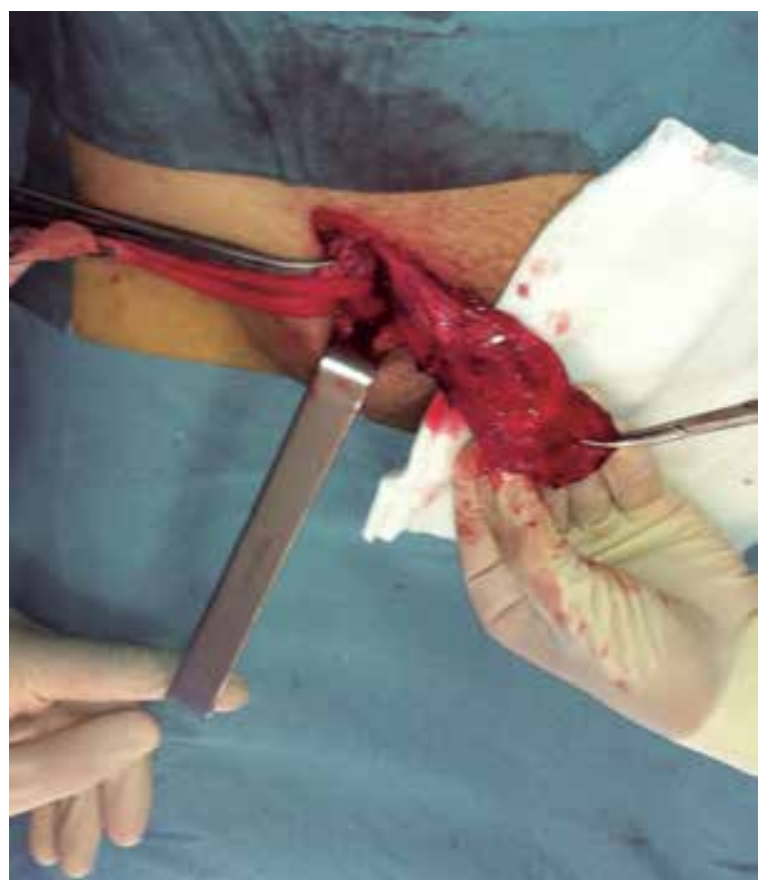

Figura 3 - Isolamento do saco herniário com o cateter no seu interior.

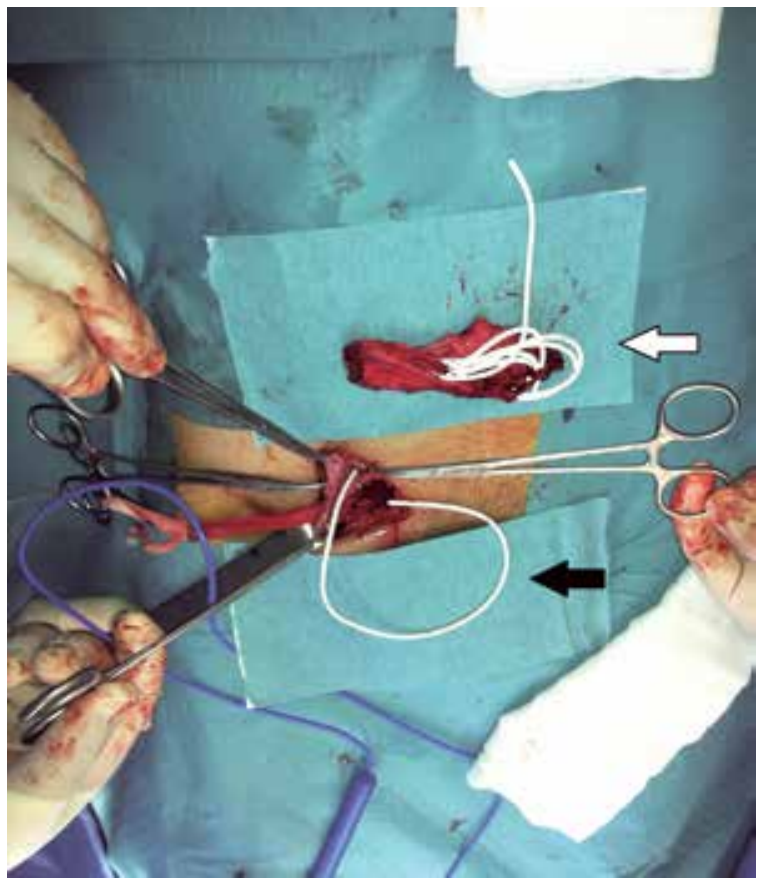

Figura 4 - Após remoção do saco herniário, removeu-se o cateter desconectado (seta branca) e foi verificado o correto funcionamento do cateter abdominal (seta negra) que se encontrava também dentro do saco herniário.

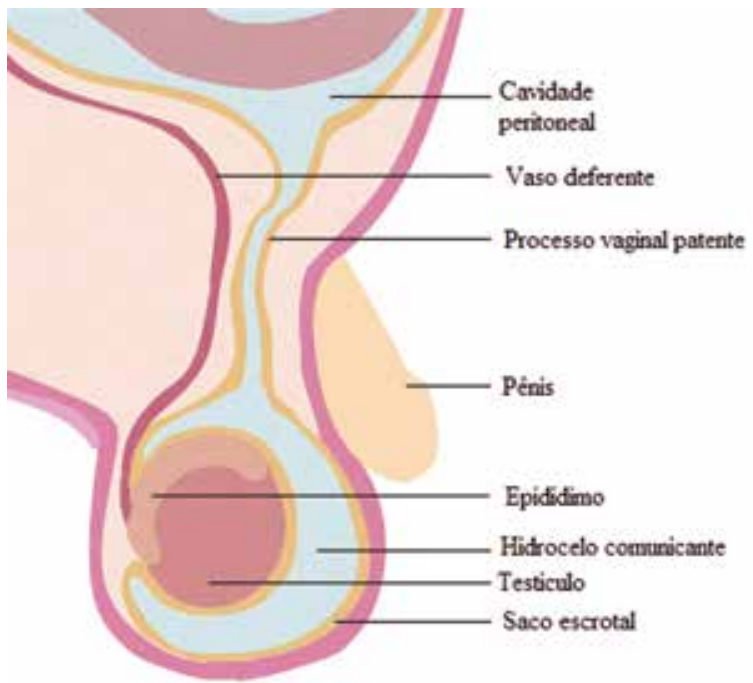

Figura 5 - A patência do processo vaginal é condição essencial para a migração do cateter para a bolsa escrotal. Nessa figura é possível perceber a comunicação da cavidade peritoneal através do processo vaginal patente.

\section{Discussão}

A patência do processo vaginal (PV) é condição anatômica necessária para a formação de hérnia inguinal ${ }^{11}$ (Figura 5). A formação de hérnia inguinal associada ao shunt ventriculoperitoneal é mais comum na infância, tendo em conta a maior taxa de patência do PV nos primeiros meses de vida (até $80 \%$ no recém-nascido). No adulto, o PV está patente em cerca de $15 \%$ a $30 \% .^{12,13}$

A idade em que a inserção da DVP é realizada poderá estar relacionada com o aparecimento de hérnias inguinais, havendo relatos de casos desde recém-nascidos até os 7 anos de idade. ${ }^{14}$ No adulto, não há estudo que correlacione uma maior taxa dessa complicação com a colocação de DVP. Para que a migração do cateter para o saco escrotal ocorra, é necessário que o PV esteja patente,$^{11} \mathrm{e}$ a presença de hérnia inguinal seria um facilitador para que esse processo aconteça. Não foi encontrado relato de migração do cateter para o saco escrotal em adulto. Uma explicação para a menor incidência no adulto pode estar relacionada com o fato de o cateter ocupar um espaço proporcionalmente menor dentro da cavidade peritoneal do adulto, primeiro porque a cavidade abdominal é proporcionalmente maior em relação ao cateter, e depois porque a maioria dos neurocirurgiões corta o excesso do cateter distal, uma vez que não há preocupação com o crescimento do doente. Obviamente que o fato de o cateter estar desconectado facilitou a sua migração através da hérnia inguinal.

No doente em questão, após revisão do processo clínico, não foi encontrado relato de hérnia ou tumefação na região inguinal antes da colocação da DVP. Mesmo assim, não é possível afirmar que haja relação entre a 
colocação de DVP e o aparecimento de hérnia inguinal. Apesar de não haver sinais de complicação pela hérnia em si, optamos por uma rápida correção cirúrgica, tendo em conta o contexto de um doente que estava cada vez menos colaborante, mais agitado e com idas frequentes à urgência por motivo de dor na região inguinal.

\section{Conflito de interesses}

Os autores declaram não haver conflito de interesses.

\section{Referências}

1. Sells CJ, Loeser JD. Peritonitis following perforation of the bowel: a rare complication of a ventriculoperitoneal shunt. J Pediatr. 1973;83(5):823-4.

2. Wilson $\mathrm{CB}$, Bertan V. Perforation of the bowel complicating peritoneal shunt for hydrocephalus. Report of two cases. Am Surg. 1966;32(9):601-3.

3. Wakai S. Extrusion of a peritoneal catheter through the abdominal wall in an infant. Case report. J Neurosurg. 1982;57(1):148-9.

4. Patel $\mathrm{CD}$, Matloub $\mathrm{H}$. Vaginal perforation as a complication of ventriculoperitoneal shunt. Case report. J Neurosurg. 1973;38(6):761-2.

5. Fukamachi A, Wada H, Toyoda O, Wakao T, Kawafuchi J. Migration or extrusion of shunt catheters. Acta Neurochir (Wien). 1982;64(1-2):159-66.

6. Ho CC, Jamaludin WJ, Goh EH, Singam P, Zainuddin ZM. Scrotal mass: a rare complication of ventriculoperitoneal shunt. Acta Medica (Hradec Kralove). 2011;54(2):81-2.
7. Ozveren MF, Kazez A, Cetin H, Ziyal IM. Migration of the abdominal catheter of a ventriculoperitoneal shunt into the scrotum - case report. Neurol Med Chir (Tokyo). 1999;39(4):313-5.

8. Clarnette TD, Lam SK, Hutson JM. Ventriculo-peritoneal shunts in children reveal the natural history of closure of the processus vaginalis. J Pediatr Surg. 1998;33(3):413-6.

9. Grosfeld JL, Cooney DR. Inguinal hernia after ventriculoperitoneal shunt for hydrocephalus. J Pediatr Surg. 1974;9(3):311-5.

10. Celik A, Ergün O, Arda MS, Yurtseven T, Erşahin Y, Balik $E$. The incidence of inguinal complications after ventriculoperitoneal shunt for hydrocephalus. Childs Nerv Syst. 2005;21(1):44-7.

11. Albala DM, Danaher JW, Huntsman WT. Ventriculoperitoneal shunt migration into the scrotum. Am Surg. 1989;55(11): 685-8.

12. Oktem IS, Akdemir H, Koç K, Menkü A, Tucer B, Selçuklu $A$, et al. Migration of abdominal catheter of ventriculoperitoneal shunt into the scrotum. Acta Neurochir (Wien). 1998;140(2):167-70.

13. Rowe MI, Copelson LW, Clatworthy HW. The patent processus vaginalis and the inguinal hernia. J Pediatr Surg. 1969;4(1):102-7.

14. Henriques JG, Pinho AS, Pianetti G. Complication of ventriculoperitoneal shunting: inguinal hernia with scrotal migration of catheter. Case report. Arq Neuropsiquiatr. 2003;61(2B):486-9.

Endereço para correspondência

Eduardo Procaci da Cunha

Largo Abel Salazar

4099-001 - Porto, Portugal

Telefone: +351 222077500, extensão 1317

E-mail: eduardopcunha@gmail.com 